\title{
Immune-Enhancing Effect of Kombucha on Cyclophosphamide-induced Immunosuppressive Mice
}

\author{
${ }^{a, b}$ Chunlong Wang , ${ }^{a, b}$ Mengying $X u,{ }^{a, b}$ Shan Gao , ${ }^{a b}$ Tianchen \\ Wu , ${ }^{a, b}$ Chengguang Qin , ${ }^{a, b}$ Zesheng Zhang*
}

${ }^{a}$ Tianjin University of Science \& Technology, Tianjin 300457, China

${ }^{\mathrm{b}}$ Key Laboratory of Food Nutrition and Safety, Ministry of Education, College of Food Engineering and Biotechnology, Tianjin University of Science \& Technology, Tianjin 300457, China

${ }^{*}$ Corresponding Author: Zesheng Zhang

E-mail: zhangzesheng@tust.edu.cn; 1157195738@qq.com

Keywords: Kombucha, Cyclophosphamide, Immune-Enhancing activity

\begin{abstract}
The purpose of the present study was to investigate the immune-enhancing activity of Kombucha. In immunosuppression experiment, the immune-enhancing effect of Kombucha in immunosuppressive mice was performed.It was showed that Kombucha at high and medium doses doses was able to overcome the CY-induced immunosuppression, significantly improves the body weight, the count of white blood cell, increases the thymus and spleen indices, enhances lymphocyte proliferation activity and peritoneal macrophage function, improves natural killer cells activity compared with negative control group. In addition, the different dose of Kombucha could significantly enhance the activity of alkaline phosphatase (AKP), superoxide dismutase (SOD), catalase (CAT), glutathione peroxidase (GSH-PX) in blood serum. Therefore, the Kombucha could be an effective immunomodulatory agent.
\end{abstract}

\section{Introduction}

Kombucha, also known as black tea mushroom, is a traditional folk acidic beverages that is fermented by sweetened black tea through mixed microorganism [1-3]. Fermentation broth of Kombucha contains tea, live microorganisms and their metabolic products [3]. This beverage has at least 100 years of history in China, it is also very popular in Japan, the United States, Canada and other places. Kombucha contains many beneficial compounds to human body that have been produced during fermentation. According to the report, Kombucha has positive effect on antioxidant, antibacterial, regulating gastrointestinal, the drain of cholesterol levels, and enhancing immunity. Kombucha may also serve as an effective functional beverage in enhancing body immunity [3-6].

In the past few years, many studies mainly focused on the antioxidant, antibacterial, lowering blood lipid effects of Kombucha [6, 7]. However, the immune-enhancing activity of Kombucha has been seldom reported. Therefore, the present study seeks to investigate the immune-enhancing activities of Kombucha in cyclophosphamide (CY)-induced immunosuppressed mice. The results may provide insights into the underlying mechanisms of activity.

\section{Materials and methods}

\subsection{Materials and chemicals}

Sugar, tea, and culture of Kombucha were provided by Tianjin Qijun Biotechnology Co., Ltd. Cyclophosphamide (CY) was purchased from Jiangsu Hengrui Medicine Co. (Lianyungang, Jiangsu, China). RPMI 1640, 3-(4,5-Dimethylthiazol-2-yl)-2,5-diphenyltetrazolium bromide (MTT), Dimethyl sulfoxide (DMSO), $0.1 \%$ neutral red staining solution, red blood cell lysis buffer, lipopolysaccha-ride (LPS) and Concanavalin A (ConA) were purchased from Solarbio (Science \& Technology Corporation, Beijing, China). Astragalus essence oral liquid was purchased from 
Yangtze River Pharmaceutical (Group) Co., Ltd. Assay kits for alkaline phosphatase (AKP), superoxide dismutase (SOD), catalase (CAT), glutathione peroxidase (GSH-PX) were all obtained from Nanjing Jiancheng Bioengineering Institute (Nanjing, Jiangsu Province, China) and all the other chemicals used in the present study were of analytical grade.

\subsection{Preparation of Kombucha}

8 grams of black tea was added to a litre of water, and boiled for 100 minutes. Then the solution was filtered by a sterile sieve, adding water to $1 \mathrm{~L}$ line. 56 grams sugar was added to it. The solution was added into 5 flasks ( $250 \mathrm{~mL}$ ) and sterilized at $121^{\circ} \mathrm{C}$ for $20 \mathrm{~min}$. When it was cooled to room temperature, the Kombucha was added into flasks $(10 \% \mathrm{v} / \mathrm{v})$. After that, the flask was cultured by shaking bed at $30{ }^{\circ} \mathrm{C}$ for 4 days. Fermentation broth were frozen at $-50{ }^{\circ} \mathrm{C}$ to $-60{ }^{\circ} \mathrm{C}$ before subjecting to lyophilization. The lyophilized product of Kombucha was stored at $-20{ }^{\circ} \mathrm{C}$.

\subsection{Experimental animals}

The experiment was conducted on Male BALB/c mice (a total of 72 mice) at aged 8 weeks old (20 \pm 2 g), which were purchased from the Tianjin Hematonosis Hospital, (Tianjin, China). The mice were housed in the cages in the SPF Laboratory Animal room of Tianjin University of Science and Technology, ambient temperature is $22 \pm 2{ }^{\circ} \mathrm{C}$ and relative humidity is $40-60 \%$ with $12 \mathrm{~h}-$ light/12 h-dark cycle. The mice were fed pellet diet and water ad libitum. The animals were approved by the Animal Care and Use Committee, and all of the animal facilities and experimental procedures were carried out according to the Technical Standards for Testing \& Assessment of Health Food (2003).

After acclimatized to environment for 1 week, these mice were randomly divided into six groups (12 for each). Immunosuppressive mice were established by intraperitoneally injection of 80 $\mathrm{mg} / \mathrm{Kg} / \mathrm{BW} / \mathrm{d}$ cyclophosphamide (CY) for 3 days. Normal control group, negative control group and positive control group were treated with physiological saline solution and astragalus essence oral liquid (80 mg/Kg/BW/d); the experiment groups were treated with Kombucha for successive 12 days. Three experimental groups were treated with the lyophilized product of Kombucha at low (2 mg/kg/d), medium (4 mg/kg/d) and high (8 mg/kg/d) does, respectively.

\subsection{Determination of organ index and white blood cell count}

\subsubsection{Determination of organ index}

The mice were weighted and fasted overnight before the end of the experiment. The spleen, liver and thymus were excised after sacrificed, the spleen and thymus were weighted after being washed with physiological saline solution and dried with filter paper. The spleen and thymus index were calculated according to the formula:

Organ index $(\mathrm{mg} / \mathrm{g})$ = weight of organ / body weight.

\subsubsection{Determination of white blood cell count}

$20 \mu \mathrm{L}$ blood was added to $380 \mu \mathrm{L}$ white blood cell diluent $(1.5 \mathrm{~mL}$ Glacial acetic acid was mixed with $1 \mathrm{~mL} 1 \%$ Gentian Violet then adjusted to $100 \mathrm{~mL}$ ) to calculated the white blood cell count by a blood count board [8].

\subsection{Effect of macrophage phagocytic function on neutral red}

All the operations were performed in aseptic condition. The mice were cervical decapitation soaked in $75 \%$ alcohol for 5 min after sacrificed. $10 \mathrm{~mL}$ cold PBS was injected into the abdominal cavity, the peritoneal fluid were sucked out to sterile tube after kneaded abdomen for 1-2 min. The peritoneal fluid was centrifuged at $1000 \mathrm{r} / \mathrm{min}$ for $5 \mathrm{~min}$ at $4{ }^{\circ} \mathrm{C}$ and the peritoneal macrophages were collected. The cells were suspended in RPMI 1640 medium, which was supplemented with $10 \%$ new-born bovine serum, to a final density of $2 \times 106$ cells $/ \mathrm{ml}$. Macrophages were cultured in $5 \%$ CO2 atmosphere at $37^{\circ} \mathrm{C}$ for 3 hours in a 96 -well plate $(100 \mu \mathrm{L} /$ well $)$. The supernatant could be discarded after the cells attached was observed under an inverted microscope. $0.1 \%$ neutral red dye solution was added into each well and cultivated 3 hours. The excess of neutral red dye was removed by washing with PBS for 3 times and the cell lysate was added into each well (100 $\mu \mathrm{L} /$ well), overnight at $4{ }^{\circ} \mathrm{C}$. The absorbance at $540 \mathrm{~nm}$ was measured on enzymatic-reader. The phagocytic activity of peritoneal macrophages in mice as calculated according to the following formula: 


\section{$\alpha=$ OD experimental well - OD zero adjusting well}

\subsection{The Measurement of natural killer (NK) cells activity}

The spleens was gently grinded and crushed on stainless steel screen (200 mesh) then homogenized with RPMI640 medium. The single-cell suspension was collected according to a previous method $[9,10]$. The red blood cells were removed by the addition of red blood cell lysis buffer (1 mL) and incubation at room temperature for 3-5 min, the erythrocyte lysis reaction was terminated by the addition of $1 \mathrm{~mL}$ new-born bovine serum, the splenic lymph was collected after a centrifugation at $1500 \mathrm{r} / \mathrm{min}$ for $10 \mathrm{~min}$. The splenic lymph and murine liver cancer cell were suspended in RPMI 1640 medium supplemented with $10 \%$ new-born bovine serum to a final density of $2 \times 106$ cells $/ \mathrm{mL}$ and $1 \times 105$ cells/ml, respectively. In a 96-well plate, was added to target cells and effector cells $100 \mu \mathrm{L}$ each well were mixed in experiment well (OD1); was added to effector and medium $100 \mu \mathrm{L}$ were mixed in the control well of effector cells (OD2) each well; the target cells were used instead of effector in the control well of target cells (OD3). $10 \mu \mathrm{L}$ of MTT (5 $\mathrm{mg} / \mathrm{mL}$ ) were added to culture solution and cultured in $5 \% \mathrm{CO} 2$ atmosphere at $37{ }^{\circ} \mathrm{C}$ for 4 hours. The supernatant was discarded after centrifuged at $1000 \mathrm{r} / \mathrm{min}$ for $10 \mathrm{~min}, 100 \mu \mathrm{L}$ of DMSO was added and shaken fully until the crystals dissolved. The absorbance at $570 \mathrm{~nm}$ was measured on enzymatic-reader. The natural killer (NK) cells activity as calculated according to the following formula:

\subsection{Detection of lymphocyte proliferation activity of the spleen}

Preparation method of spleen cells with 2.6. The cells were suspended to a final density of 2×107 cells/ml in RPMI 1640 medium supplemented with 10\% new-born bovine serum and added $100 \mu \mathrm{L}$ each well in a 96-well plate respectively in accordance with the method. Each mice was set up the control group, the group of $\mathrm{T}$ lymphocytes activated by ConA and the group of $\mathrm{B}$ lymphocytes activated by LPS, all determinations were conducted in triplicate. The control group was added to RPMI-1640 medium and spleen cells, $100 \mu \mathrm{L} /$ well; the group of $\mathrm{T}$ lymphocytes activated by ConA was added to ConA solution and spleen cells; the group of B lymphocytes activated by LPS was added to LPS solution and spleen cells. And then cultured in 5\% CO2 atmosphere at $37^{\circ} \mathrm{C}$ for 40 hours, after that cultured 4 hours with $10 \mu \mathrm{L} 5 \mathrm{mg} / \mathrm{mL}$ MTT each well. The supernatant was discarded, added DMSO $150 \mu \mathrm{L}$ each well and shaken fully until the crystals dissolved. The absorbance was measured on enzymatic-reader at $570 \mathrm{~nm}$. The stimulation index (SI) cells activity as calculated according to the following formula:

$\mathrm{SI}=\mathrm{OD}$ control group / OD experiment group

\subsection{Detection of blood serum biochemical indicators in mice}

The mice pick eye take blood, the sample was centrifuged at $3000 \mathrm{r} / \mathrm{min}$ for $10 \mathrm{~min}$ at $4{ }^{\circ} \mathrm{C}$, after that the supernatant was stored in the $-20^{\circ} \mathrm{C}$ refrigerator to be measured. The kits instructions were followed to detect enzyme activities.

\subsection{Statistical analysis}

All data were analysed using statistical software SPASS19.0 and the difference was considered significantly if $\mathrm{p}<0.05$. The single factor analysis of variance by ANOVA and multiple comparison test by Duncans. The data was presented as mean \pm standard deviation.

\section{Results}

\subsection{Effects of Kombucha on the body weight}

A reduced immune regulation level caused by CY resulted in the loss of body weight. 
Table 1 Changes in the body weight of every group

\begin{tabular}{lll}
\hline Groups & Initial $(\mathrm{g})$ & Final $(\mathrm{g})$ \\
\hline Normal control & $24.01 \pm 2.24^{\mathrm{a}}$ & $25.94 \pm 1.79^{\mathrm{a}}$ \\
Negative control & $25.65 \pm 3.06^{\mathrm{a}}$ & $18.76 \pm 2.33^{\mathrm{c}}$ \\
Positive control & $24.19 \pm 2.40^{\mathrm{a}}$ & $23.41 \pm 1.89^{\mathrm{b}}$ \\
Low dose & $25 \pm 2.51^{\mathrm{a}}$ & $21.93 \pm 2.81^{\mathrm{b}}$ \\
Medium dose & $25.59 \pm 2.13^{\mathrm{a}}$ & $22.38 \pm 1.98^{\mathrm{b}}$ \\
High dose & $23.72 \pm 2.29^{\mathrm{a}}$ & $22.39 \pm 2.58^{\mathrm{b}}$ \\
\hline
\end{tabular}

Data are expressed as mean \pm standard deviation $(n=12)$. Means not sharing a common superscript a $-\mathrm{c}$ differ significantly at $\mathrm{p}<0.05$.

As shown in Table 1, the body weight of negative control group significantly decreased $(\mathrm{p}<0.05)$, compared with normal control group. The mice treated with Kombucha exhibited significantly increases $(p<0.05)$ on body weight compared with negative control group. The experiment groups performed no significant enhancement compared with positive control group $(p>0.05)$.

\subsection{The changes of organ index}

The effects of Kombucha on the organ index were showed in Fig.1.

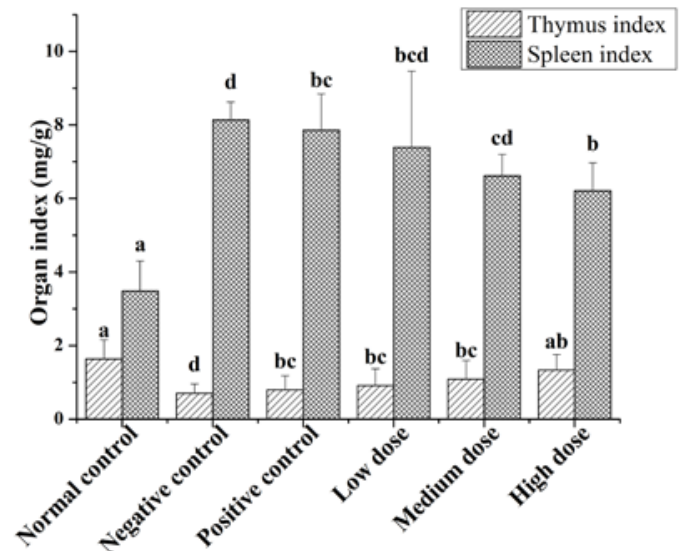

Figure. 1 The changes of thymus and spleen index. Data are expressed as mean \pm standard deviation $(n=12)$. Means not sharing a common superscript a $-d$ differ significantly at $p<0.05$.

In normal control group, the levels of thymus index and spleen index have a significant increase and decrease $(\mathrm{p}<0.05)$, compared with negative control group. Thymus index were significantly increased $(p<0.05)$ in high dose group, whereas other groups treated with Kombucha exhibited no significantly increase compared with negative control group. Spleen index were significantly decreased $(\mathrm{p}<0.05)$ in medium dose and high dose group compared with negative control group. The organ index in positive control group changed no significant difference was observed in positive control group.

\subsection{Effect of Kombucha on white blood cell amount in CY-induced immunosuppressive mice}

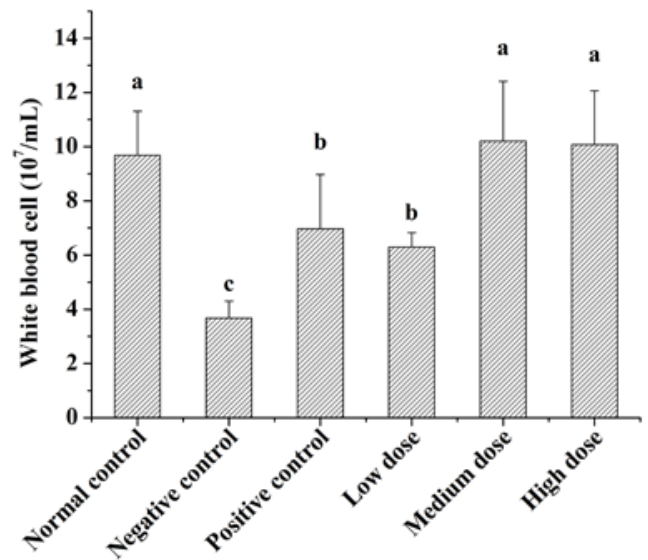

Figure.2 Effect of Kombucha on white blood cell amount. Data are expressed as mean \pm standard deviation $(\mathrm{n}=12)$. Means not sharing a common superscript a - e differ significantly at $\mathrm{p}<0.05$. 
It was shown in Fig.2, compared with normal control group, the white blood cell amount of negative control group significantly decreased $(p<0.05)$, indicated that the hematopoietic system and immune cells were damaged. There was a significant decrease $(p<0.05)$ in amount of white blood cell of positive control, Kombucha-treated groups compared with negative control group. The white blood cell amount in immunosuppressive mice treated with Kombucha (medium and high dose group) were significantly higher compared with positive control group. Fig.2a illustrated that the Kombucha played a certain role in the recovery on immune function in immunosuppressive mice induced by cyclophosphamide.

\subsection{Effect of Kombucha on phagocytosis of peritoneal macrophages in CY-induced} immunosuppressive mice

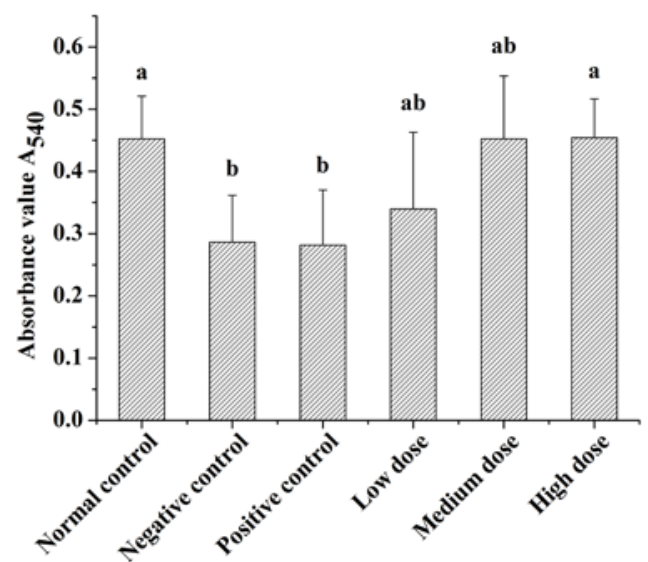

Figuer.3 Effect of Kombucha on phagocytosis of peritoneal macrophages. Data are expressed as mean \pm standard deviation $(n=12)$. Means not sharing a common superscript a - e differ significantly at $p<0.05$.

Fig.3 illustrated that the absorbance value level were significantly decreased in negative control group ( $\mathrm{p}<0.05$ ), compared with normal control group. However, the absorbance level were significantly increased in high dose group $(\mathrm{p}<0.05)$ compared with negative control group. But the phagocytosis of peritoneal macrophages in positive control group exhibited no significant difference compared with negative control group $(p>0.05)$. The results showed that Kombucha enhanced the phagocytic activity of peritoneal macrophages in CY-induced immunosuppressive mice.

\subsection{Effect of Kombucha on NK cells cytotoxicity in CY-induced immunosuppressive mice}

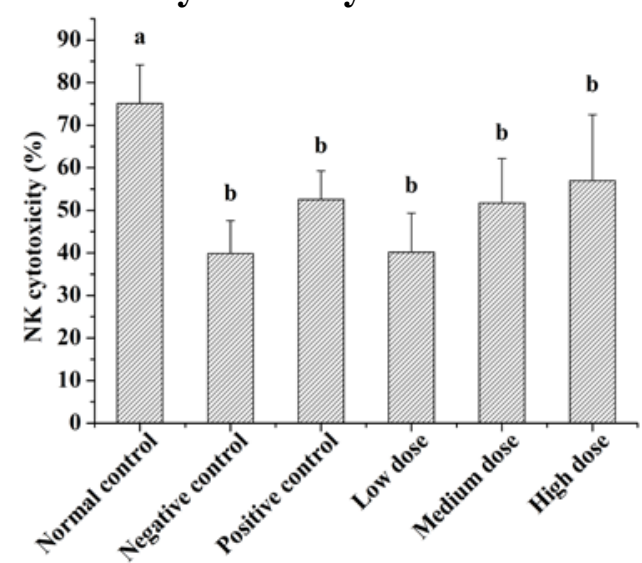

Figure.4 Effect of Kombucha on activity of natural killer cells and the proliferation level of splenic lymphocyte in CY-induced immunosuppressive mice. Data are expressed as mean \pm standard deviation $(\mathrm{n}=12)$. Means not sharing a common superscript a - e differ significantly at $p<0.05$.

NK cells cytotoxicity in CY-induced immunosuppressive mice were showed in Fig.4. Compared with normal control group, NK cells cytotoxicity of negative control group was significant decreased $(\mathrm{p}<0.05)$, indicated that the model of immunosuppression was established. NK cells cytotoxicity in Kombucha-treated groups were significantly improved in a dose dependent manner. 
Compared with negative and positive control group. These results indicated that Kombucha strengthened the activity of natural killer cells on hepatocellular carcinoma.

\subsection{Effect of Kombucha on splenocyte proliferation in CY-induced immunosuppressive mice}

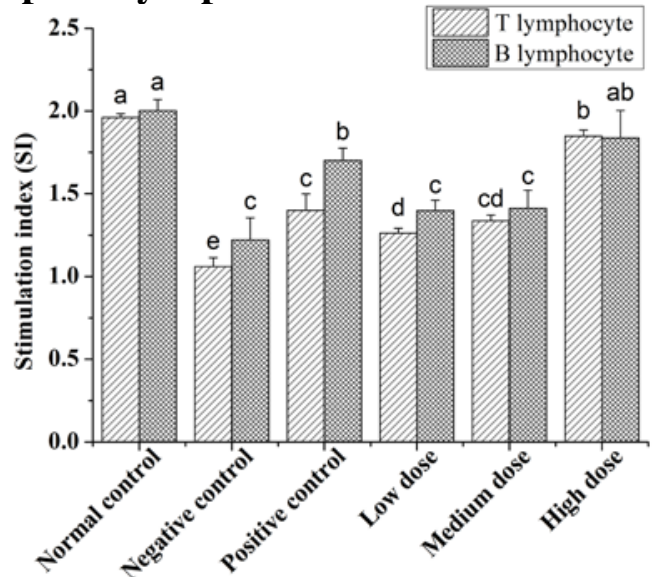

Figure.5 Effect of Kombucha on the proliferation level of splenic lymphocyte in CY-induced immunosuppressive mice. Data are expressed as mean \pm standard deviation $(n=12)$. Means not sharing a common superscript a - e differ significantly at $p<0.05$.

The effects of Kombucha on the proliferation levels of T lymphocyte and B lymphocyte were showed in Fig.5. Compared to normal control group, negative control was significantly decreased (p $<0.05$ ). This proliferation levels of T lymphocyte and B lymphocyte in Kombucha-treated groups was found to be increased ( $\mathrm{p}<0.05$ ) compared with negative control group. Meanwhile, the experiment groups were increased in a dose-dependent manner compared with positive control group. These results indicated that the immune-enhancing effect of Kombucha may be related to its enhancement on proliferation levels of T lymphocyte and B lymphocyte.

\subsection{Effect of Kombucha on enzymatic activity of blood serum in CY-induced} immunosuppressive mice.

Table 2 Effect of Kombucha on GSH-PX, AKP, SOD and CAT of blood serum in CY-induced immunosuppressive mice

\begin{tabular}{lllll}
\hline Groups & $\begin{array}{l}\text { GSH-PX } \\
(\mathrm{U} / \mathrm{mg})\end{array}$ & $\begin{array}{l}\text { AKP } \\
(\text { King unit } / \mathrm{g})\end{array}$ & $\begin{array}{l}\text { SOD } \\
(\mathrm{U} / \mathrm{mg})\end{array}$ & $\begin{array}{l}\text { CAT } \\
(\mathrm{U} / \mathrm{mg})\end{array}$ \\
\hline Normal control & $456.21 \pm 53.03^{\mathrm{a}}$ & $23.48 \pm 3.36^{\mathrm{a}}$ & $158.63 \pm 16.16^{\mathrm{a}}$ & $13.09 \pm 1.93^{\mathrm{a}}$ \\
Negative control & $371.32 \pm 19.36^{\mathrm{c}}$ & $12.58 \pm 1.67^{\mathrm{c}}$ & $107.50 \pm 27.76^{\mathrm{c}}$ & $9.99 \pm 1.31^{\mathrm{b}}$ \\
Positive control & $383.95 \pm 26.77^{\mathrm{bc}}$ & $17.62 \pm 2.09^{\mathrm{b}}$ & $141.59 \pm 18.81^{\mathrm{ab}}$ & $14.10 \pm 2.06^{\mathrm{a}}$ \\
Low dose & $376.24 \pm 29.62^{\mathrm{c}}$ & $13.78 \pm 2.12^{\mathrm{c}}$ & $123.94 \pm 11.74^{\mathrm{bc}}$ & $15.51 \pm 0.75^{\mathrm{a}}$ \\
Medium dose & $394.21 \pm 25.65^{\mathrm{bc}}$ & $16.25 \pm 1.86^{\mathrm{b}}$ & $144.19 \pm 29.54^{\mathrm{ab}}$ & $13.96 \pm 1.74^{\mathrm{a}}$ \\
High dose & $415.37 \pm 25.42^{\mathrm{b}}$ & $17.02 \pm 1.50^{\mathrm{b}}$ & $155.59 \pm 15.61^{\mathrm{a}}$ & $15.15 \pm 1.58^{\mathrm{a}}$ \\
\hline
\end{tabular}

Data are expressed as mean \pm standard deviation $(\mathrm{n}=12)$. Means not sharing a common superscript a $-\mathrm{c}$ differ significantly at $\mathrm{p}<0.05$

The activities of GSH-PX, AKP, SOD and CAT of blood serum were presented in Table 2. Compared to normal control group, negative control was significantly decreased ( $p<0.05)$. The results showed that the activities of GSH-PX, AKP, SOD and CAT of blood serum in Kombucha treated groups were improved to different degrees compared with negative control group $(\mathrm{p}<0.05)$.

\section{Discussion}

Kombucha is a beverage that contains many beneficial substances. It is confirmed that Kombucha has a variety of effects on the human health. In the present study, we have attempted to illustrate efficacy of Kombucha in CY-induced immunosuppressive mice.

The white blood cell is an important part of the body's defence system, which includes all kinds of granulocyte, lymphocyte and single cells. The effect of alkylation on cyclophosphamide and 
DNA cells, formation of cross links can inhibit growth of tumour cell. In addition, this effect can inhibit and damage the hematopoietic system and immune cells, resulting in the declined of white blood cell count and immune function reaction [8]. This study found that different dose of Kombucha could enhance the white blood cell count respectively, indicated that Kombucha can inhibit the damage of cyclophosphamide on hematopoietic system and immune cells, eliminate invading pathogens and allergens in different ways and mechanisms, and regulate the body's immune function.

The spleen and thymus are important immune organs in mammals[11]. Spleen and thymus indexes can directly reflect the level of immune functions in the body, and effects of drugs on the spleen index and thymus index can be used as the preliminary indicators for the study on immunopharmacological mechanisms in animals[12]. The weight of thymus and spleen reflected the immune level in a certain extent. After mice was injected CY, their immune function was damaged. Compared to thymus involution and thymus index decreased, spleen enlargement and spleen index increased. The reason might be that the immune function of mice was inhibited, and the spleen compensatory increased so that the spleen index increased. This indicated that Kombucha could protect the thymus and spleen in CY-induced immunosuppressive mice, thereby enhanced the function of body's immune system.

Macrophages could be involved in nonspecific immunity, humoral immunity and cellular immunity, it also could be used as antigen-presenting cells. Activated macrophages not only participate in both specific immune and non-specific immune reactions but also serveas the "bridge cell" of these two types of immune reactions [13]. The phagocytosis of macrophage was an important indicator that could measure the level of humoral immunity [14]. The results showed that Kombucha could enhance the immune function by improving the phagocytic activity of peritoneal macrophage on cell debris and pathogens.

Natural killer cells is one of the most important immune cells existing in the spleen, it is neither require specific participation, nor need non-specific antigen sensitized killer cells. The main target of natural killer cells are cells injected by virus tumour cells, autologous cells and parasites, so it is an important part of anti-tumour and anti-infection system to humoral immunity [9]. The results showed that the different doses of Kombucha could increase the ability of natural killer cells against hepatocellular carcinoma in a dose-dependently manner.

The spleen is not only an important site of immune response, but also the largest immune organ. The proliferation of $\mathrm{T}$ and $\mathrm{B}$ lymphocyte are simulated with ConA and LPS as mitogen. After antigen is stimulated, $\mathrm{T}$ and $\mathrm{B}$ lymphocyte could produce specific immune response, it is called antigen specific lymphocyte or immunocompetent cells. In the immune response, it is the mutual cooperation and restriction between all kinds of lymphocyte and subsets that completed the identification, response and elimination of hazardous and alien substance, maintaining the internal environment stable [15]. Results showed that treatment with Kombucha could promote the recovery of splenocyte proliferative responses to both $\mathrm{T}$ and $\mathrm{B}$ lymphocytes in $\mathrm{CY}$-treated mice organisms and that this response is dose-dependent. Compared with the result of phagocytosis of peritoneal macrophages, NK cells cytotoxicity and proliferation levels of lymphocyte, we could see that Kombucha stimulated macrophages and $\mathrm{T}$ lymphocytes to release cytokines, which effected on natural killer cells and enhanced its activity. This result was consistent with Ishikawa's [16].

GSH-Px is an enzyme that can reduce toxic peroxides to hydroxyl compounds with low toxicities to protect cells from being damage of the peroxides to maintain the structure and function of normal cells $[17,18]$. SOD can reduce the level of intracellular superoxides by catalyzing the conversion of superoxide radicals to molecular oxygen and peroxide hydrogen [19, 20]. AKP is an important enzymes of metabolic regulation in organism that can be directly involved in the physiological process of transfer and metabolism of phosphate groups, it also plays an important role in immune reaction in human body. CAT is one of the key enzyme to scavenge oxygen free radicals in the body's defence system that can cause disproportionation decomposition of H2O2, protecting cells from being damaged by the oxygen free radicals, and reducing the cell apoptosis induced by oxidative stress [21]. The results showed that the different dose of Kombucha could significantly 
enhance the activity of GSH-PX, SOD, AKP and CAT. In addition, Kombucha could alleviate the body injury of CY-induced immunosuppressive mice by enhancing the activity of antioxidant enzyme and reducing the damage of immune system by oxidative stress.

\section{Conclusion}

The daily administration of hygienic conditioned Kombucha to CY-induced experimental mice shows pronounced beneficial effects as immune as it shows significant increased on the levels of the body weight, the count of white blood cell, the organ index of thymus and spleen, the phagocytosis of peritoneal macrophages, natural killer cells cytotoxicity and the proliferation level of splenic lymphocyte which was decreased up on cyclophosphamide (CY) treatment and also significantly reversed the altered the enzyme activities of GSH-PX, SOD, AKP and CAT. The present study gives an indication that Kombucha can enhance the ability of body immunity in CYinduced immunosuppressive mice, and can effectively resist the aging and disease caused by oxidative stress. Meanwhile, the effect of Kombucha on enhancing immunity significantly higher than the effect of astragalus essence oral liquid. The precise mechanism and detailed composition of Kombucha are still to be determined, to promote Kombucha as an effective functional food in the prevention and treatment of immune diseases.

\section{Acknowledgement}

This work was found by the project supported by the National Science-technology pillar program (2012BAD33B05) and the Ministry of education of the People's Republic of China's project (Grant No. IRT1166).

\section{Reference}

[1] H. Battikh, A. Bakhrouf, Ammar, E., Antimicrobial effect of Kombucha analogues. Lwt-Food Sci Technol 2012, 47, 71-77.

[2] S. C. Chu, C. S. Chen, Effects of origins and fermentation time on the antioxidant activities of kombucha. Food Chem 2006, 98, 502-507.

[3] C. Dufresne, E. Farnworth, Tea, Kombucha, and health: a review. Food Res Int 2000, 33, 409421.

[4] A. J. Marsh, O. O'Sullivan, C. Hill, R. P. Ross, P. D. Cotter, Sequence-based analysis of the bacterial and fungal compositions of multiple kombucha (tea fungus) samples. Food Microbiol 2014, 38, 171-178.

[5] T. Srihari, K. Karthikesan, N. Ashokkumar, U. Satyanarayana, Antihyperglycaemic efficacy of kombucha in streptozotocin-induced rats. J Funct Foods 2013, 5, 1794-1802.

[6] A. S. Velicanski, D. D. Cvetkovic, S. L. Markov, V. T. T. Saponjac, J. J. Vulic, Antioxidant and Antibacterial Activity of the Beverage Obtained by Fermentation of Sweetened Lemon Balm (Melissa officinalis L.) Tea with Symbiotic Consortium of Bacteria and Yeasts. Food Technol Biotech 2014, 52, 420-429.

[7] M. L. Watawana, N. Jayawardena, C. Choo, V. Y. Waisundara, Application of the Kombucha 'tea fungus' for the enhancement of antioxidant and starch hydrolase inhibitory properties of ten herbal teas. Food Chem 2016, 194, 304-311.

[8] A. V. Gadow, E. Joubert, C. F. Hansmann, Comparison of the Antioxidant Activity of Aspalathin with That of Other Plant Phenols of Rooibos Tea (Aspalathus linearis), $\alpha$-Tocopherol, BHT, and BHA. Journal of Agricultural \& Food Chemistry 1997, 45, 632-638.

[9] C. Korzeniewski, D. M. Callewaert, An enzyme-release assay for natural cytotoxicity. Journal of Immunological Methods 1983, 64, 313-320. 
[10] K. Joachim, S. Alexander, W. Carsten, M. Ofer, Activating natural cytotoxicity receptors of natural killer cells in cancer and infection. Trends in Immunology 2013, 34, 182-191.

[11] S. Venkatramanujam, S. R. Pandi-Perumal, B. Amnon, K. P. Bhatnagar, D. P. Cardinali, Melatonin, immune function and cancer. Recent Patents on Endocrine Metabolic \& Immune Drug Discovery 2011, 5, 109-123(115).

[12] B. Chen, L. Liu, H. Xu, Y. Yang, Effectiveness of immune therapy combined with chemotherapy on the immune function and recurrence rate of cervical cancer. Experimental \& Therapeutic Medicine 2015, 9, 1063-1067.

[13] S. Gordon, The role of the macrophage in immune regulation. Research in Immunology 1998, 149, 685-688.

[14] A. Bielak-Zmijewska, M. Koronkiewicz, J. Skierski, K. Piwocka, Effect of curcumin on the apoptosis of rodent and human nonproliferating and proliferating lymphoid cells. Nutrition \& Cancer 2000, 38, 131-138.

[15] T. A. Fleisher, J. B. Oliveira, Functional and molecular evaluation of lymphocytes. Journal of Allergy \& Clinical Immunology 2004, 114, 227-234.

[16] I. Hideki, S. Tomoko, O. Toru, S. Takaichiro, Aged garlic extract prevents a decline of NK cell number and activity in patients with advanced cancer. Journal of Nutrition 2006, 136, 816S-820S.

[17] B. C. Wilke, M. Vidailhet, A. Favier, C. Guillemin, Selenium, glutathione peroxidase (GSH-Px) and lipid peroxidation products before and after selenium supplementation. Clinica Chimica Acta 1992, 207, 137-142.

[18] S. Bhattacharya, R. Gachhui, P. C. Sil, Effect of Kombucha, a fermented black tea in attenuating oxidative stress mediated tissue damage in alloxan induced diabetic rats. Food Chem Toxicol 2013, 60, 328-340.

[19] X. W. Zhou, X. F. Wang, Q. Z. Li, Expression and characteristic of the Cu/Zn superoxide dismutase gene from the insect parasitizing fungus Cordyceps militaris. Mol Biol Rep 2012, 39, 10303-10311.

[20] H. Chao, A. J. Ryan, M. A. Shubha, C. Brent, Accelerated development of pulmonary fibrosis via $\mathrm{Cu}, \mathrm{Zn}$-superoxide dismutase-induced alternative activation of macrophages. Journal of Biological Chemistry 2013, 288, 20745-20757.

[21] G. Elizabeth, K. Kevin, H. Kelly, R. Julianna, Increased microglial catalase activity in multiple sclerosis grey matter. Brain Research 2014, 1559, 55-64. 